\title{
A novel approach to characterising quantum theory based on limited information and complementarity
}

\author{
Philipp Hoehn* \\ Perimeter Institute for Theoretical Physics \\ E-mail: phoehnaperimeterinstitute.ca
}

\begin{abstract}
The last decade has seen a wave of reconstructions and characterizations of quantum theory using the formalism of generalized probability theory. In this talk, we shall outline a novel (operational) approach to characterizing and reconstructing quantum theory which gives primacy to limited information and complementarity rather than the probability structure. In particular, we consider an observer interrogating a system with binary questions and analyze the consequences of (1) a postulate asserting a limit on the (simultaneous) information the observer can acquire about the system, and (2) a postulate asserting the existence of complementarity on the set of possible questions. We explain how the ensuing compatibility and complementarity structure of the binary questions implies many features of qubit quantum theory in an elegant way (e.g. threedimensionality of the Bloch sphere, the entanglement structure of two qubits, absence of third or higher order interference and many other features). Time permitting, we shall also sketch how this program can be completed to a full reconstruction of quantum theory by adding further ingredients.
\end{abstract}

Frontiers of Fundamental Physics 14 - FFP14,

15-18 July 2014

Aix Marseille University (AMU) Saint-Charles Campus, Marseille

\footnotetext{
*Speaker.
} 